\title{
X-RAY MEASUREMENT OF STRESSES IN DEPOSITED THIN FILM WITH FIBER TEXTURE
}

\author{
Takao HANABUSA
}

\author{
Faculty of Engineering, Tokushima University \\ 2-1, Minamijosanjima, Tokushima 770-8506, Japan
}

\begin{abstract}
Thin film coating by physical vapor deposition (PVD) and chemical vapor deposition (CVD) techniques are advancing intensively in the technology of mechanical as well as electrical fields. Deposition by these techniques essentially develops residual stresses in the film for intrinsic and extrinsic reasons. The microstructure of the film normally exhibits a strong preferred orientation on a crystallographic scale. Residual stresses and the microstructure of the growing film influence the properties of the film as well as the film/substrate system. The present paper demonstrates the recent developments in X-ray stress evaluation of the film having a very sharp preferred orientation. A new method of X-ray stress measurement for such films having a strong texture is presented in place of the conventional $\sin ^{2} \Psi$ method. C-axis oriented aluminum nitride (AIN) films, [111] fiber textured aluminum and titanium nitride (TiN) films and [110] fiber textured TiN films are investigated to study the residual stress and the effect of heat treatment on the state of residual stress. The effects of patterning the film on residual stress are also investigated.
\end{abstract}

Key words: $X$-ray stress measurement, Thin film, Residual stress, Thermal stress, c-axis orientation, Fiber texture, AlN film, Al film, TiN film

\section{INTRODUCTION}

$\mathrm{X}$-ray stress measuring technique is a very effective non-destructive tool to measure residual and applied stresses in polycrystalline materials with sufficient reliability. Usual $\mathrm{X}$-ray stress evaluation is based on the $\sin ^{2} \Psi$ method $[1,2]$ by which the lattice strains at various angles of $\psi$, the angle between the normal of a diffraction plane and the normal of a specimen surface, linearly depend on $\sin ^{2} \psi$. This relation is based on the assumptions that the materials consist of randomly orientated small crystals and that the plane stress state exists in the X-ray diffracting region. With these assumptions, diffraction intensities and therefore lattice strains can be measured at any $\psi$-angle. From the strains for various $\psi$-angles we can construct a $2 \theta($ or $d)$ - $\sin ^{2} \psi$ diagram for evaluating the stress occurring in the $X$-ray diffracting region, where $2 \theta$ is the diffraction angle and $d$ is the lattice spacing evaluated by Bragg's law.

If a material has some textures, the diffraction intensity is not uniform in relation to $\psi$ and intensity peaks appear at particular $\psi$ depending on the textures of the specimen [3-7]. An extreme case of texture can be seen in the films deposited by PVD (physical vapor deposition) and CVD (chemical vapor deposition). In a case that hcp (hexagonal closed packed) crystals such as AlN and $\mathrm{ZnO}$ are deposited on a substrate, the basal plane, i.e., $(00 \cdot 1)$ of the crystal, arranges itself parallel to the film surface [8-12]. When a fcc (face-centered cubic) crystal such as aluminum, titanium nitride (TiN) or titanium carbide (TiC) film is deposited on a substrate, \{111\} plane is likely to arrange itself parallel to the surface, and in some cases $\{110\}$ or $\{100\}$ lies parallel to the surface [13-19]. In such cases, the diffraction intensity cannot be observed at an arbitrary $\psi$-angle excepting certain angles. Therefore, the $\sin ^{2} \psi$ method of stress evaluation for randomly oriented material should be modified for precise measurement of stresses in textured thin films.

\section{STRESS MEASUREMENT OF HCP THIN FILM WITH C-AXIS ORIENTATION}

An aluminum nitride (AIN) film is a promising candidate for a high frequency surface acoustic wave device because of its high acoustic velocity [20,21]. A thin film of AlN has been grown on a variety of substrates by several techniques [22-25]. Among many techniques, a plasma magnetron sputtering method with two facing targets [26] produces a well-oriented crystal structure suitable for the surface acoustic wave (SAW) device. Besides the film structure, residual stresses developed in the film are an important factor for the reliability of thin films. Residual stresses in the film may sometimes be the cause of film cracking or delamination from the substrate. Therefore, the evaluation and then the control of residual stresses are very important to make reliable AlN films.

Figure 1 shows the diffraction pattern of the AlN film on a BLC (borosilicate) glass substrate, which was obtained with CrKa characteristic X-rays at $\Psi=0 \mathrm{deg}$ [8]. Compared with the pattern obtained from a powder sample (Fig. 2), the only the peak which appears is 00.2 diffraction at $2 \theta=54.7 \mathrm{deg}$.

It means that $(00 \cdot 1)$ plane of the crystals in the film lies parallel to the surface of substrate, i.e., c-axis of

Received March 18, 1999

Accepted May 13, 1999 


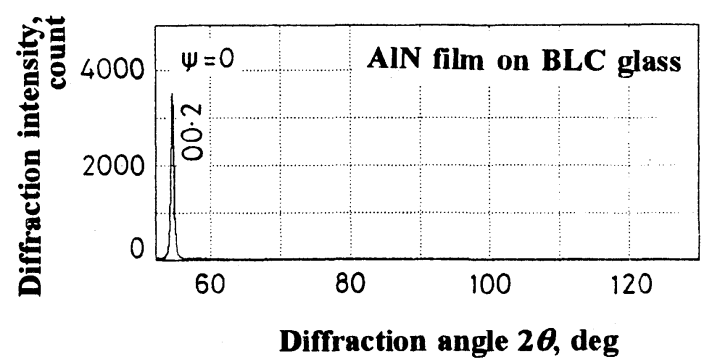

Fig. 1. Diffraction pattern measured on AlN film deposited on glass substrate [8].

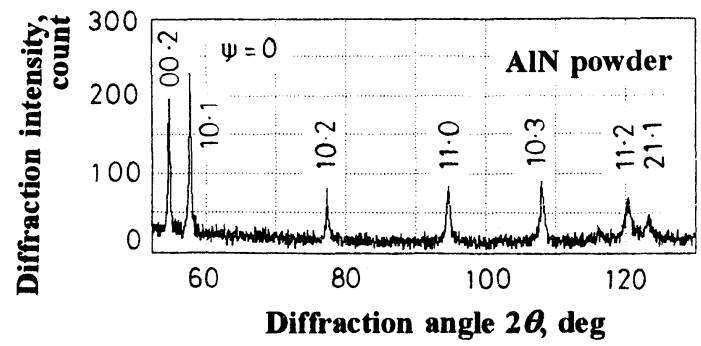

Fig. 2. Diffraction pattern measured on AlN powder sample [8].
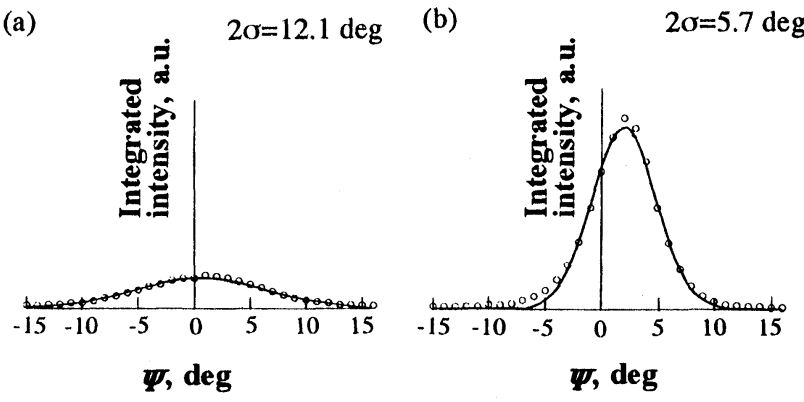

Fig. 3. $\Psi$-angle dependency of $00 \cdot 2$ diffraction from AlN film deposited at (a) $373 \mathrm{~K}$ and (b) $523 \mathrm{~K}$ [9].

AlN crystals arranges along the surface normal. More precise measurement of preferred orientation is given in Fig. 3, which indicates a distribution of $00 \cdot 2$ diffraction intensity of AlN film against $\boldsymbol{\psi}$-angle [8]. The observable intensity is restricted within a region of $\pm 15 \mathrm{deg}$ in $\psi$ and there are no signals beyond this region. Twice the standard deviation of the Gaussian fitting curves on the data points, $2 \sigma$, which is defined as a c-axis orientation, is 12.1deg for the AlN film deposited at $373 \mathrm{~K}$ and $5.7 \mathrm{deg}$ for the one at $523 \mathrm{~K}$. This means that the c-axis orientation deteriorates in the film at low substrate temperatures, but improves in the film at high substrate temperatures. The broadening of these $I-\psi$ curves shows that the c-axis of all the crystals does not perfectly arrange itself along the normal of the film surface, but actually fluctuates from the surface normal.

As shown in these figures, the crystals in the deposited film are not randomly oriented; therefore the socalled $\sin ^{2} \psi$ method cannot be adopted in the present

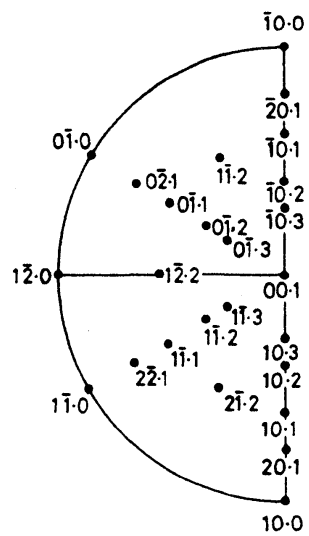

(a)

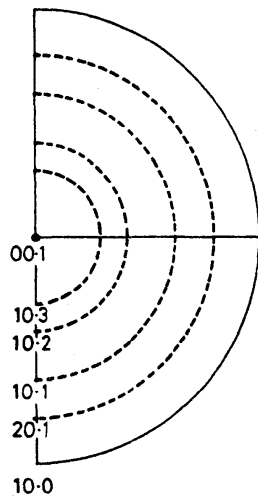

(b)
Fig. 4. Stereographic projection of hcp crystal: (a) standard $(00 \cdot 1)$ projection for AlN and (b) projection for AlN powder sample [8].

Table 1. Diffraction planes and related parameters.

\begin{tabular}{lccc}
\hline$h k \cdot l$ & $2 \theta(\operatorname{deg})$ & $\psi(\operatorname{deg})$ & $\sin ^{2} \psi$ \\
\hline $00 \cdot 2$ & 54.7 & 0.0 & 0.00 \\
$10 \cdot 1$ & 57.3 & 61.6 & 0.77 \\
$10 \cdot 2$ & 61.6 & 42.3 & 0.42 \\
$10 \cdot 3$ & 107.6 & 31.6 & 0.28 \\
\hline
\end{tabular}

case. The author and co-workers presented a new analyzing method [8], which is briefly explained in the following.

Figure 4 (a) shows a standard stereographic projection of a hcp single crystal where various directions of lattice planes are represented by small dots (poles). In a case of the film having a sharp preferred orientation, we usually observe a columnar structure in a cross-sectional view and an aggregation of tiny crystals in a planar view of the film. From these observations, it seems that each crystal has a random orientation around c-axis with $2 \pi$ rotational freedom. Therefore, the stereographic projection of the film can be represented by a rotation of the standard stereographic projection around $00 \cdot 1$ pole, as shown in Fig. 4(b).

When the X-ray irradiation inclines from the surface normal, we successively encounter different diffraction peaks at any azimuth, for example, 10.3, 10.2 and 10.1, at proper angles defined by the angular difference between the normal of each $(h k \cdot l)$ plane and the $<00 \cdot 1>$ direction. As shown in Fig. 4, the basal plane $(00 \cdot 1)$ is parallel to the film surface; its higher order diffraction $00 \cdot 2$ is therefore measured as in Fig. 1.

Table 1 shows the diffraction plane used in the stress measurement, their Bragg angles, their observable $\psi$ angles and the values of $\sin ^{2} \psi$. Figure 5 shows examples of the measured profile diffracted from the $h k \cdot l$ plane indicated in Table 1. The intensity of diffraction from 00.2 is very strong because all crystals contribute to the diffraction. On the other hand, other three diffraction peaks are weak since crystals which contribute to them take up only a small part in the whole ring of the ster- 


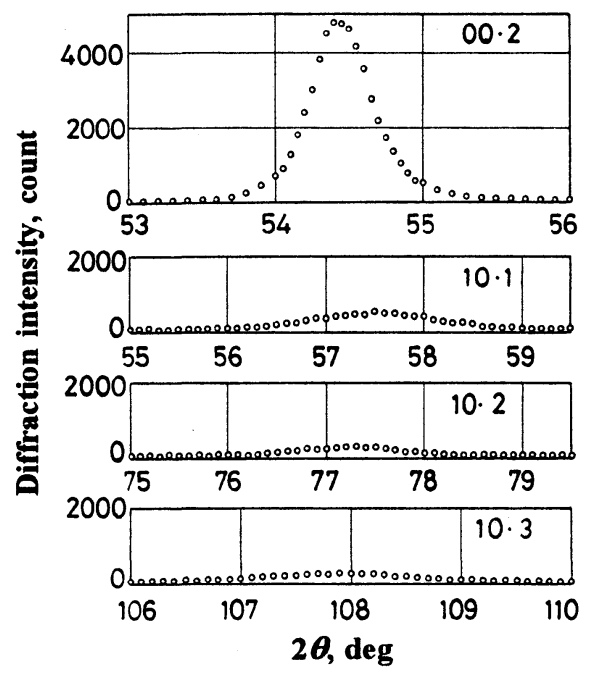

Fig. 5. Diffraction profiles measured on AlN film [8].
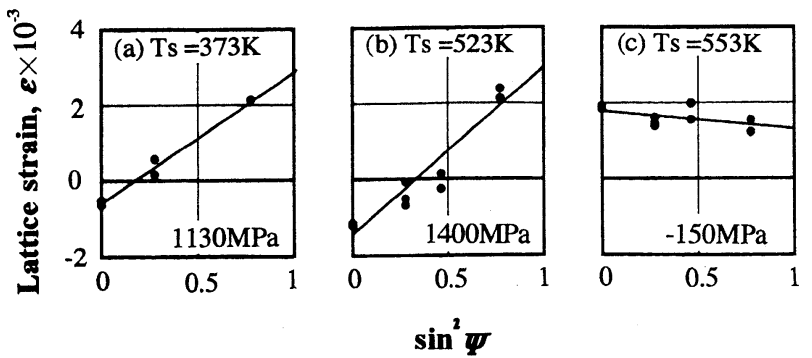

Fig. 6. Relation between lattice strains and $\sin ^{2} \psi$ obtained from AlN films [9].

eographic projection of the film sample. For such small intensity, the Gaussian fitting method [27] becomes extremely useful to determine the precise position of the diffraction line.

In general, the lattice strain $\varepsilon_{33}^{L}$ in any orientation is related to the stress components $\sigma^{8}{ }_{y}$ in the coordinate system attached on a specimen by the following equation:

$$
\varepsilon^{\mathrm{L}}{ }_{33}=\gamma_{31} \gamma_{3 j} \pi_{m k} \pi_{n t} \boldsymbol{S}^{*}{ }_{j H} \sigma_{m n}^{\mathrm{s}}=s^{\mathrm{L}}{ }_{33 m n} \sigma_{m n}^{\mathrm{s}}
$$

where $s^{*}$ are elastic compliances of a single crystal and $\gamma_{v}$ and $\pi_{i v}$ are the components of the transformation matrices from the crystal system to the laboratory system and from the crystal system to the specimen system, respectively.

If we assume that the stress state is equi-biaxial, i.e., $\sigma_{11}^{\mathrm{s}}=\sigma_{22}^{\mathrm{s}}=\sigma^{\mathrm{s}}$ and other $\sigma^{\mathrm{s}}{ }_{i}$ are zero, Eq. (1) is then reduced to

$$
\varepsilon^{L}{ }_{33}=\left\{\left(s^{*}{ }_{11}+s^{* 2}-2 s^{*}{ }_{13}\right) \sin ^{2} \Psi+2 *^{*}{ }_{13}\right\} \sigma^{3}
$$

where $s_{i j}$ are elastic compliances of the single crystal in a matrix style and $\psi$ is, in the present case, equal to the angle between $(00 \cdot 1)$ plane and each $(h k \cdot l)$ plane to be measured.

Equation (2) means that the lattice strain for each $(h k \cdot l)$ plane relates linearly to $\sin ^{2} \Psi$. Therefore, if we could measure a set of $h k \cdot l$ diffraction, the strains for each diffraction plane should lie on a straight line. From a slope of the line determined by the least square method, and given the value of $\left(s^{*}{ }_{11}+s^{*} 22-2 s^{*}{ }_{13}\right)$, stress value can be estimated by the following relation:

$$
\sigma=\left(s^{*}{ }_{11} s^{*}{ }_{22}-2 s^{*}{ }_{13}\right)^{-1} \frac{\partial \varepsilon^{L}{ }^{2}}{\partial \sin ^{2} \psi}
$$

where the values of $s^{*}{ }_{v}$ for AlN crystal are; $s^{*}{ }_{11}=0.35$, $s^{*}{ }_{22}=-0.20$ and $2 s^{*}{ }_{13}=0.30 \times 10^{-5} / \mathrm{MPa}$.

It is important to note that not a single type of diffraction line but various types are used in this stress analyzing method. These diffraction lines appear at proper $\psi$-angles determined by the crystal structure as shown in Table 1 . By the conventional $\sin ^{2} \psi$ method, the diffraction line used in a series of measurement is one type, $\{h k l\}$. In this case, the stress-free $d$-spacing $\left(d_{0}\right)$ or $2 \theta$ value $\left(2 \theta_{0}\right)$ is not always necessary to analyze $\mathrm{X}$-ray stress. As long as we use different types of $h k l$ diffraction, however, it is important to know a stress-free $d_{0}$ or $2 \theta_{0}$ value of each diffraction line so as to calculate a normalized lattice strain. This point is entirely different from the $\sin ^{2} \psi$ method.

As already shown in Table 1 , the diffraction lines available for the measurement lie in a wide range from $54 \mathrm{deg}$ to $107 \mathrm{deg}$ within $2 \theta$. AlN powder is used as a standard material to eliminate errors arising from any misalignment of the stress measuring system. At the geometrically determined $\boldsymbol{\psi}$-angle for each diffraction (see Table 1), the position of diffraction line for the powder and that of the film specimens can be measured. Then, the lattice strain is calculated by the following equation:

$$
\varepsilon(h k \cdot l, \Psi)=\frac{d_{\mathrm{f}}(h k \cdot l, \Psi)-d_{\mathrm{p}}(h k \cdot l, \Psi)}{d_{\mathrm{p}}(h k \cdot l, \Psi)}
$$

where $d_{\mathrm{r}}$ and $d_{\mathrm{p}}$ are the interplanar spacings measured from the film and the powder, respectively.

Figure 6 shows examples of correlation between lattice strain and $\sin ^{2} \psi$ measured from the film deposited at the substrate temperatures of $373 \mathrm{~K}, 523 \mathrm{~K}$ and $553 \mathrm{~K}$. A fairly good linear relation can be observed in spite of very weak diffraction excepting at $\boldsymbol{\psi}=0 \mathrm{deg}$. The former two cases (a) and (b) each have a steep positive gradient, which means tensile stress, but the latter one (c) has a small negative gradient. From the gradient of these lines the stress value can be estimated by Eq. (3).

Figure 7 shows the results of the stress measurement of AIN films. Tensile stresses were detected in the films on both BLC glass and quartz substrates. A large tensile residual stress of $1 \sim 2 \mathrm{GPa}$ was found in the films deposited below $523 \mathrm{~K}$ and the stress value increased as the substrate temperature increased from $373 \mathrm{~K}$ to $523 \mathrm{~K}$. The residual residual stress itself and its increment in the films deposited on quartz were much larger than those on the BLC glass. This is reflected by different thermal expansion coefficients between BLC and quartz substrates. The thermal expansion coefficients of BLC glass, quartz, and AlN in the basal plane are $5.1 \times 10^{-6} / \mathrm{K}$, 


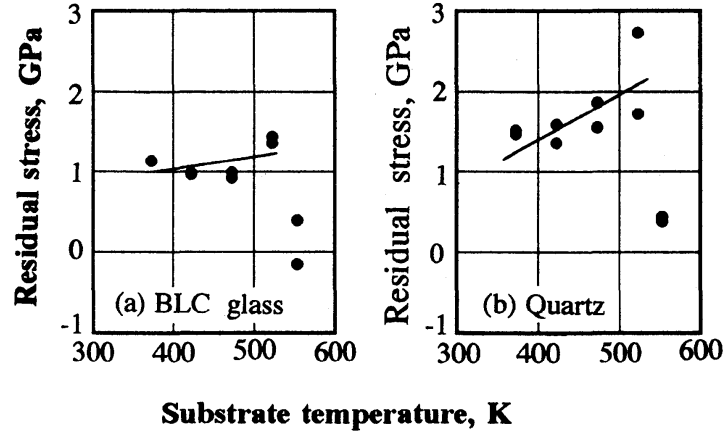

Fig.7. Dependence of substrate temperature on residual stress in AlN films deposited on (a) BLC glass and (b) quartz substrate [9].

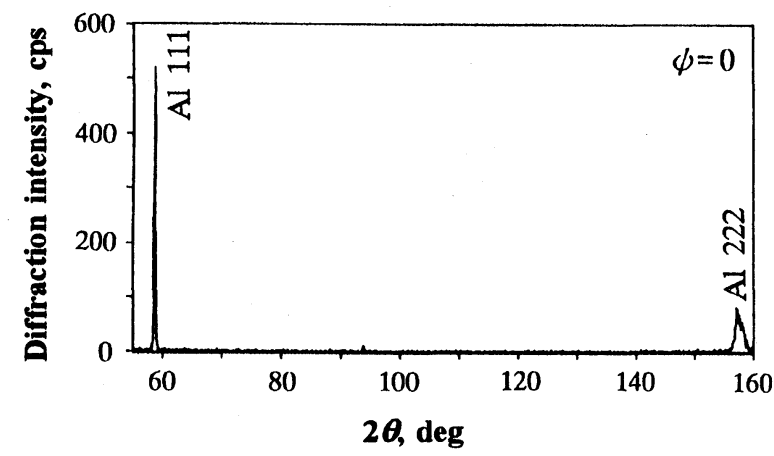

Fig. 8. Diffraction pattern measured on $\mathrm{Al}$ film on silicon wafer [13].

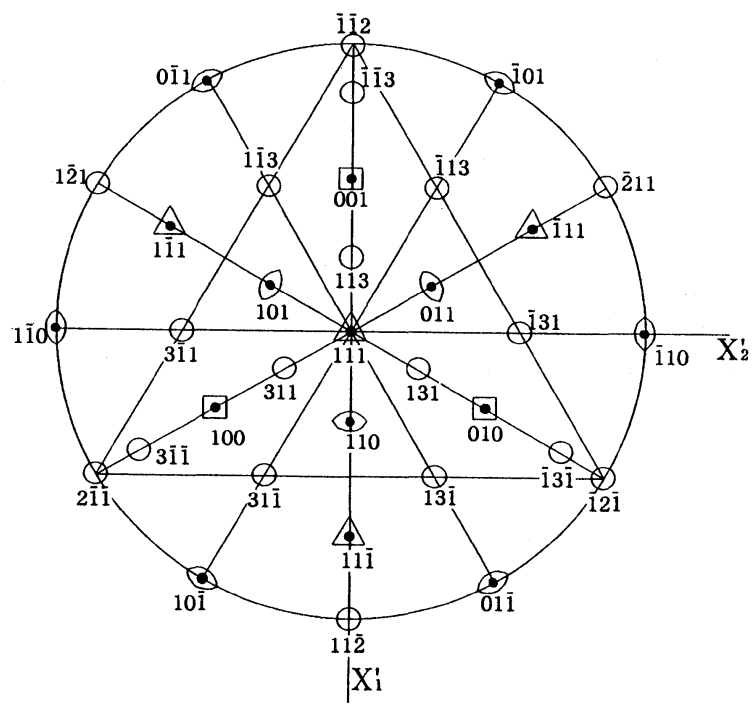

Fig. 9. (111) stereographic projection of cubic crystal [31].

$5.6 \times 10^{-7} / \mathrm{K}$ and $5.6 \times 10^{-6} / \mathrm{K}$, respectively. The thermal stress which occurs by the differences in thermal contraction rates between different materials when they are cooled from the depositing temperature to the room temperature is calculated by the following equation;

$$
\sigma=\frac{E_{\text {film }}}{1-v_{\text {film }}} \Delta a \Delta T
$$

where $E_{\text {film }}$ is Young's modulus and $\nu_{\text {flm }}$ is Poisson's ratio of the film, $\Delta a=a_{\text {film }}-a_{\text {ab }}$ where $a_{\text {film }}$ and $\alpha_{\text {ab }}$ are the thermal expansion coefficients of the film and the substrate, respectively, $\Delta T$ is the temperature drop from the depositing state to the room temperature. In the present case, $\Delta a$ is negligible for the BLC glass substrate system but large for the quartz substrate system. Since $\sigma$ depends linearly on $\Delta T$, the thermal stress increases with increasing depositing temperatures, as is shown in the case of the quarts substrate. The measured tensile stresses below $523 \mathrm{~K}$ of the substrate temperature (depositing temperature) are much higher than the stress calculated by Eq. (5). The intrinsic stress caused by reasons other than the thermal stress must contribute to a large part to the development of the residual stress. However, at higher substrate temperatures, 543K and $553 \mathrm{~K}$, the residual stress relaxes to a smaller value, probably because of sufficient crystallization and the suitable arrangement of crystal orientation so as to eliminate intrinsic stresses.

\section{STRESS MEASUREMANT OF FCC THIN FILM WITH SPECIAL ORIENTATION}

An aluminum thin film on a silicon wafer [13-15,28,29] produced by sputtering, a chromium film on a steel substrate by electroplating and a titanium nitride thin film deposited by PVD [16-18,30] have [111] preferred orientation, i.e., the [111] direction of the crystals consisting these films coincides with the normal of the film surface. All of these crystals have a fcc structure, and its closed packed plane $\{111\}$ lies parallel to the film surface.

\subsection{Aluminum film deposited by sputtering}

Figure 8 shows a diffraction pattern obtained from an Al film deposited on a silicon substrate obtained by using $\mathrm{CrKa}$ characteristic $\mathrm{X}$-rays. In this figure we observe 111 and 222 diffraction peaks over the whole range of measured $2 \theta$. Needless to say, we would have 200,220 and 311 diffraction peaks between these lines if we provided a powder sample.

The previous section says that different types of diffraction must be used in the evaluation of stresses in the hcp films with c-axis orientation. However, we can use a single type of $h k l$ diffraction in order to evaluate the stresses of fcc films with a sharp preferred orientation, because of the high symmetry of the cubic structure. The 111 stereographic projection of a cubic crystal is given in Fig. 9. As is the same with a case of the film consisting of hcp crystal, each column of the aluminum crystal has a random orientation with each other around the normal of the film surface. The stereographic projection for the film structure can therefore be assumed to be obtained by a $2 \pi$ rotation of the pole figure for a single crystal. An experimental evidence [15] is shown in Fig. 10 where 222 and 200 poles are represented by two continuous rings around the normal of the film sur 


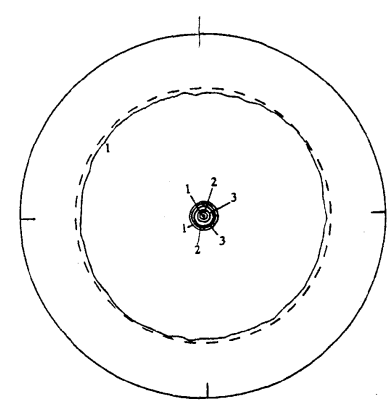

(a) 222 pole figure

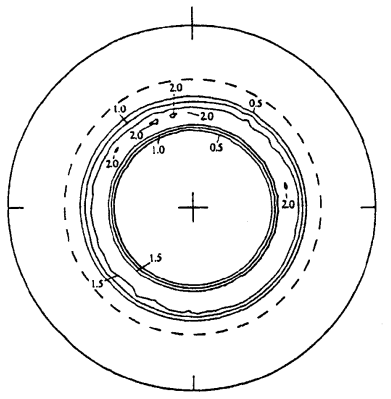

(b) 200 pole figure
Fig. 10. Pole figures of aluminum thin film deposited on silicon wafer [15].
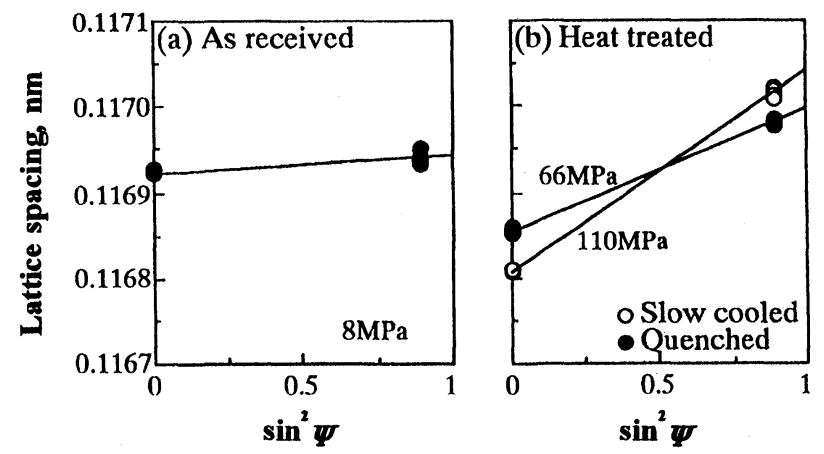

Fig. 11. Lattice spacing vs. $\sin ^{2} \psi$ relation of $\mathrm{Al}$ films for evaluating residual stresses: (a) as-received and (b) heat treated samples [13].

face, i.e., the center of the pole figure, with homogeneous intensity.

Since two $\{111\}$ planes of a cubic crystal cross each other at an angle of $70.5 \mathrm{deg}$, the intensity of 222 diffraction peaks appear at $\psi_{1}=0 \mathrm{deg}$ and $\psi_{2}=70.5 \mathrm{deg}$ for the film having 111 fiber texture. In this case the residual stress is evaluated by the following relation:

$$
\sigma=\frac{2}{s_{44}^{c}} \frac{\varepsilon_{1}^{L}-\varepsilon_{2}^{L}}{\sin ^{2} \psi_{1}-\sin ^{2} \psi_{2}}
$$

where $s^{c}$ is the elastic compliance of a single crystal, $\varepsilon_{1}^{L}$ and $\varepsilon_{2}^{L}$ are the lattice strains measured in the directions $\psi_{1}$ and $\psi_{2}$, respectively.

An example of stress measurement using the lattice spacing observed at these two $\psi$ angles is shown in Fig. 11. Figure 11(a) shows a measurement for the asreceived condition and Fig. 11(b) the data for the heat treated condition, i.e., slowly cooled and water-quenched from 473K. Small tensile residual stresses in the as- received state and large stresses in heat-treated state are observed. Because of the sharp [111] texture, the intensity of 111 diffraction is very high compared with that of the powder sample. The precision of measurment is therefore sufficiently guaranteed in spite of the two-tilt method.

Tanaka et al. [31] proposed a method for the tri-axial analysis of residual stresses in thin films having [111] fiber texture using the fundamental formulae derived on the basis of Reuss and Voigt models. By this method, strains are measured on two different types of diffraction, 222 and 311. In the case where 222 and 311 diffraction types are used to evaluate stresses in a film, the former diffraction appears at $\psi=0 \mathrm{deg}$ and $\psi=70.5 \mathrm{deg}$ and the latter at $29.5 \mathrm{deg}$ and $58.5 \mathrm{deg}$.

Supposing an equi-biaxial plane stress state, we have a relation between the strain $\varepsilon_{33}^{L}$ at each lattice plane and $\sin ^{2} \psi$ as shown in the following:

$$
\varepsilon_{33}^{L}=\frac{1}{2} s_{44} \sigma \sin ^{2} \Psi+\frac{1}{3}\left(s_{11}+4 s_{12}-s_{44}\right) .
$$

When using two types of different diffraction, $d_{0}$ value, the lattice spacing in a stress free state, is necessary to calculate normalized strain $\varepsilon$. Strains but not $d$-spacings are available for the above equation as a common index for every diffraction.

Figure 12 shows the results of film stress measurement of different thicknesses [32]. They indicate that in each graph the four measured points are aligned on a single line and that the slopes of the lines are almost identical regardless of the film thicknesses and azimuthal angles $\phi$. Another data indicates that the residual stress decreases as the film thickness increases

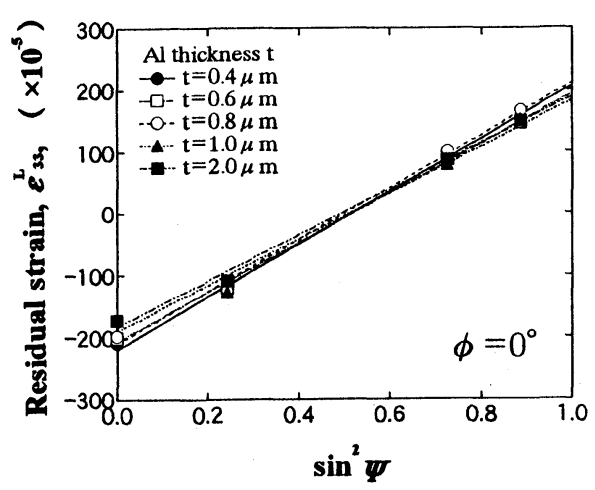

( a ) $\varepsilon_{33}^{L}\left(\phi=0^{\circ}\right)-\sin ^{2} \psi$ diagram

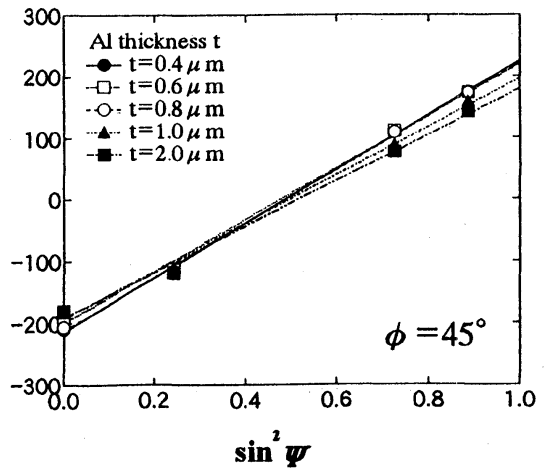

( b ) $\varepsilon_{33}^{L}\left(\phi=45^{\circ}\right)-\sin ^{2} \psi$ diagram

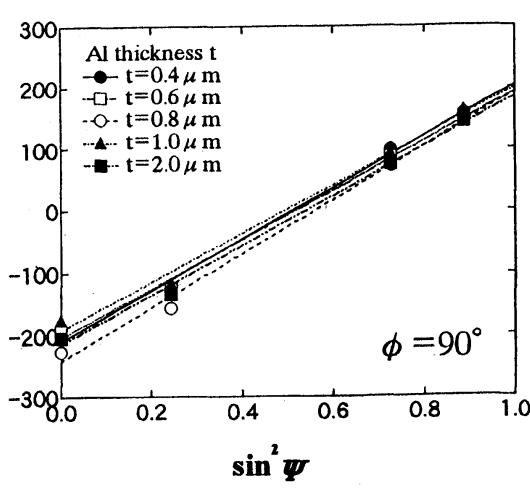

(c ) $\varepsilon_{33}^{L}\left(\phi=90^{\circ}\right)-\sin ^{2} \psi$ diagram

Fig. 12. X-ray stress measurement of $\mathrm{Al}$ film having [111] preferred orientation [32]. 

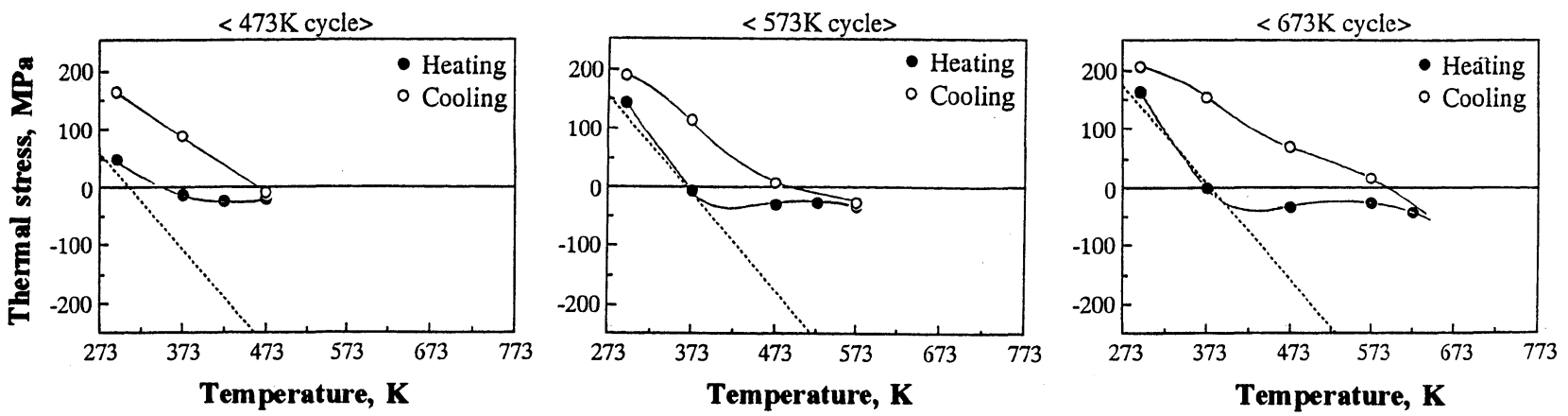

Fig. 13. In-situ thermal stress measurement of Al film without passivation during heat cycles [14].
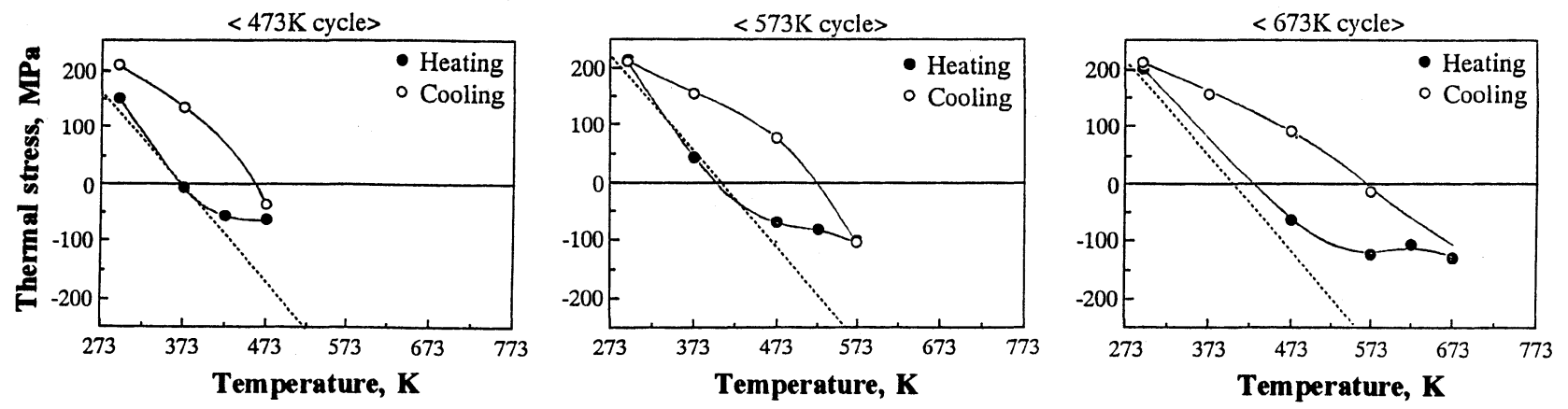

Fig. 14. In-situ thermal stress measurement of $\mathrm{Al}$ film passivated by thin AlN film during heat cycles [14].

[15]. The residual stress in a film seems to be strongly influenced by the conditions and the method of film preparation.

For simplicity, two diffraction peaks appearing at $\boldsymbol{\psi}=$ $0 \mathrm{deg}$ and $\psi=70.5 \mathrm{deg}$ are used in the stress evaluation of the film with [111] orientation. If more precise measurement is needed, it is appropriate to use 311 diffraction accompanying with the 222 diffraction. Since the lattice strains are related to Eq. (7), the stress is evaluated by the slope of the regression line, which aligns

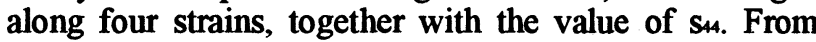
Figs. 11 and 12 it is obvious in the film deposited on a silicon wafer that the residual stress is tensile and is considered to have been derived from different thermal expansion coefficients between the aluminum film and the silicon substrate.

Figure 13 shows the results of the thermal stress measurement of an aluminum film during a series of heating and cooling cycles [13]. Figure 13(a) shows thermal stress alterations between room temperature (RT) and 473K. The initial residual stress as received condition is almost zero. As temperature increases, a small compressive stress develops from $373 \mathrm{~K}$ to $473 \mathrm{~K}$. In the cooling stage, the stress due to thermal mismatch increases almost linearly with decreasing temperatures. In the second thermal cycle between RT and 573K (Fig. 13(b)), the initial high tensile stress of $160 \mathrm{MPa}$ rapidly drops to zero at $373 \mathrm{~K}$ beyond which the rate of decrease becomes small.

The stress change $(\Delta \sigma)$ in the film due to the temperature increase $(\Delta T)$ of the aluminum film/silicon sub- strate system is calculated by the following equation:

$$
\frac{\Delta \sigma}{\Delta T}=\frac{E_{\mathrm{Al}}}{1-v_{\mathrm{Al}}} \Delta \alpha
$$

where $\Delta \alpha$ is the difference in the thermal expansion coefficients between silicon $\left(\alpha_{\mathrm{si}}=9.6^{-6} / \mathrm{K}\right)$ and aluminum $\left(a_{\mathrm{Al}}=23.9^{-6} / \mathrm{K}\right) . E_{\mathrm{Al}}(=70 \mathrm{GPa})$ is Young's modulus and $v_{\mathrm{AI}}(=0.33)$ Poisson's ratio of aluminum.

According to Eq. (8), the gradient $\Delta \sigma / \Delta T$ is -1.49 $\mathrm{MPa} / \mathrm{K}$ which is an elastic behavior in a change in thermal stresses. Although the measurement involves only two data points, the initial change in measured stresses with increasing temperature up to $373 \mathrm{~K}$ is linear with a slightly greater slope than the calculated one. Above $373 \mathrm{~K}$, the data points deviate from this elastic line, showing no significant increase in thermal stresses. This means that the aluminum film behaves in a way of a plastic flow as well as a hillock formation accompanied by atomic movements. In the cooling stage, stress initially rapidly increases to about $50 \mathrm{MPa}$, then slowing down in the next stage. The increase rate again picks up below 273K. These changes in behavior in the heating and the cooling stages are qualitatively the same in the next heat cycle between room temperature and $673 \mathrm{~K}$ as shown in Fig. 13(c). Similar results were observed both by the wafer curvature technique and the X-ray measurement [14,33-35].

Figure 14 shows a thermal stress variation of $\mathrm{Al}$ film passivated by thin AlN film. As shown in Fig. 14(a), AIN passivation creates tensile residual stress of about 

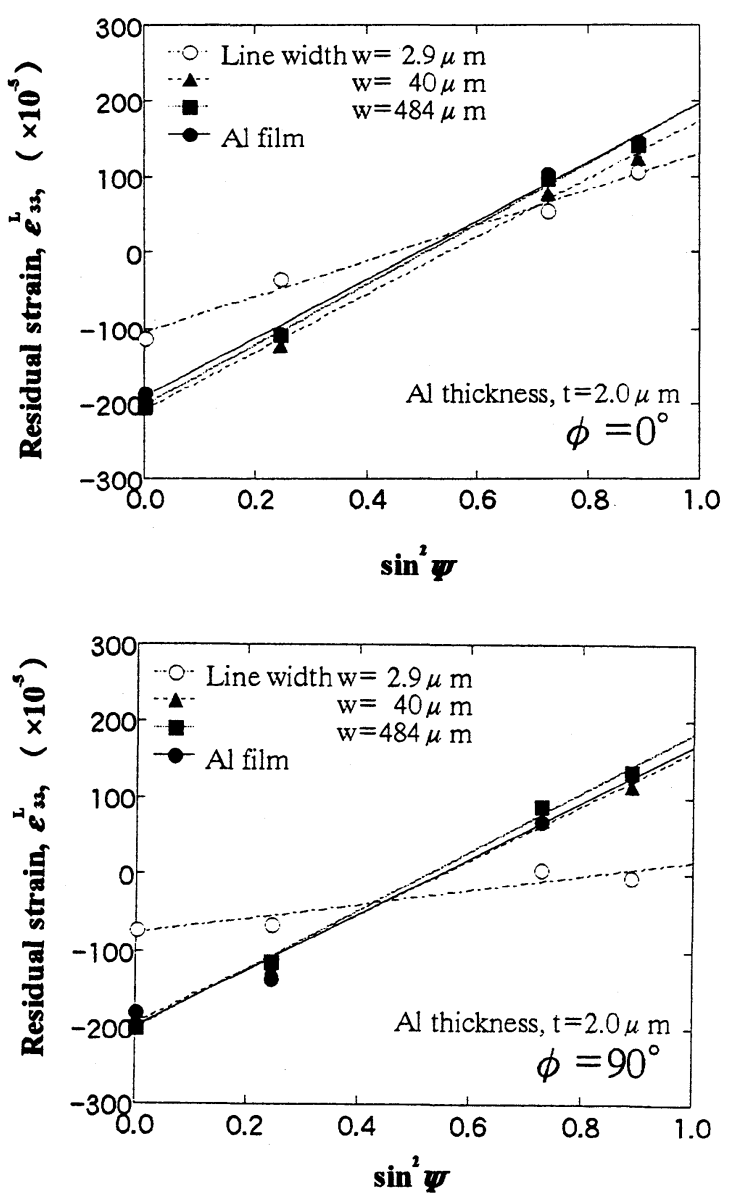

Fig. 15. Lattice strain vs. $\sin ^{2} \psi$ relation of $\mathrm{Al}$ line structures for evaluating residual stresses [38].

$150 \mathrm{MPa}$ in the aluminum film. As temperature increases the state of thermal stress approximates the slope expected by Eq. (6). When the temperature rises above $373 \mathrm{~K}$, the rate of thermal stress change becomes smaller than has been expected. However, compressive stress continues to increase and the maximum stress value attained at the terminal point of the heating cycle becomes larger than that of unpassivated aluminum film.

It is shown from Figs. 13 and 14 that residual stresses after heat cycles and the development of compressive thermal stresses in heating periods are evidently intensified by passivation. This means that the atomic movement which causes plastic flow or hillock formation is partly suppressed by the hard passivating film. It is also reported that the addition of $2.0 \mathrm{at} \% \mathrm{Ta}$ in the film enlarges the elastic region in a heating cycle due to the reduction of hillock formation. The addition of $\mathrm{Ta}$ atoms contributes to the enhancement of a high temperature strength because of the effect of solid solution [36].

We have assumed so far that the stress state in a film structure is equi-biaxial. When the film is patterned to make a line structure, the stress state is changed into non-equiaxial state $[37,38]$. Figure 15 shows the relation

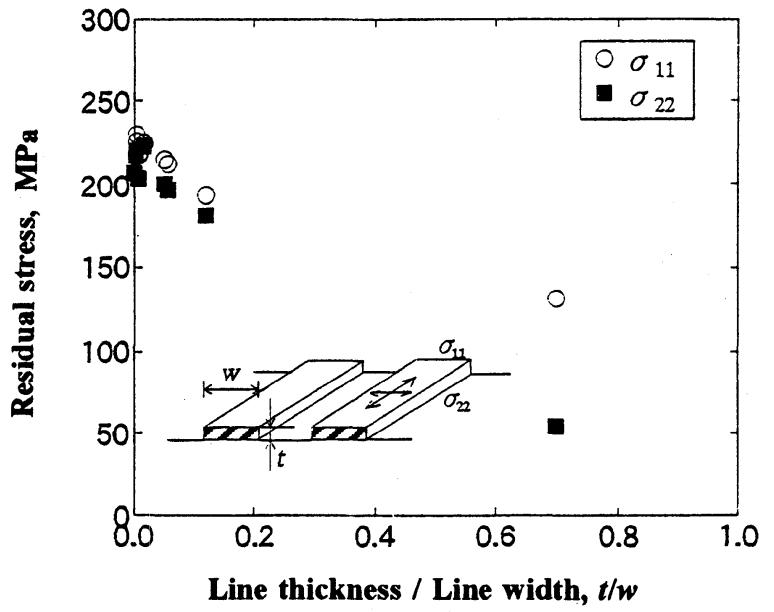

Fig. 16. Relation between residual stress and sectional shape of aluminum lines [38].

between the lattice strain and $\sin ^{2} \psi$ for $\phi=0 \mathrm{deg}$ and $90 \mathrm{deg}$ obtained from the patterned thin film, where $\phi=$ 0deg is parallel to and $\phi=90 \mathrm{deg}$ is perpendicular to the line. Figure 16 shows the results of biaxial stress state in the patterned thin film structure, indicating that the residual stresses are parallel $\left(\sigma_{11}\right)$ and perpendicular $\left(\sigma_{22}\right)$ to the patterned aluminum line as a function of the ratio of line thickness $(t)$ to line width $(w)$, under the assumption of $\sigma_{33}=0$. The residual stress in the line structure decreases with the increasing ratio $t / w$. It is also noted that $\sigma_{22}$ is much more relieved than $\sigma_{11}$ when $t / w$ becomes greater.

\subsection{TiN film deposited by PVD method}

TiN coatings are widely used as thermal corrosion barriers and for wear resistance. The use of coatings on cutting tools is most likely to improve their lives. However, residual stresses are essentially generated in coating films owing to the mismatch of thermal contraction between the film and the substrate and for other reasons [39-41]. As the residual stress may influence the mechanical properties of coated material, it is important to know the residual stress state in the film. Considerable efforts [42-47] have been made to measure the stress of

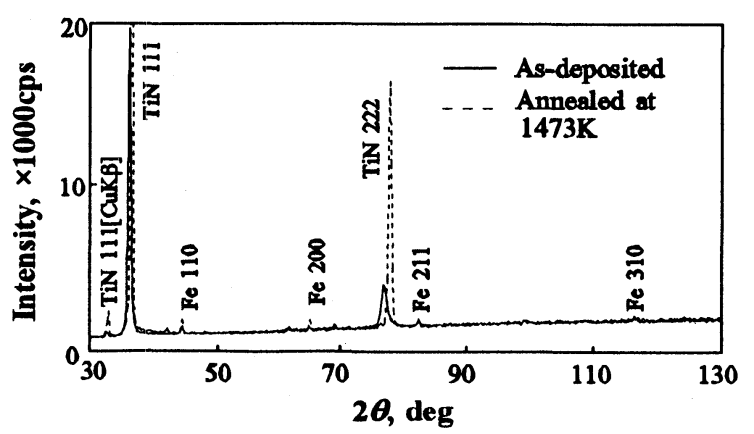

Fig. 17. Diffraction pattern measured on TiN film deposited by arc ion plating [45]. 


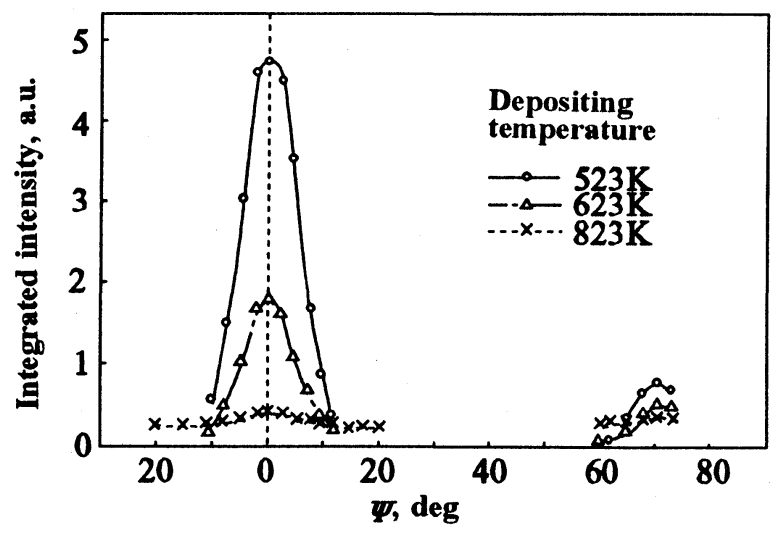

Fig. 18. $\psi$-angle dependency of 111 diffraction intensity from TiN film deposited by arc ion plating [46].

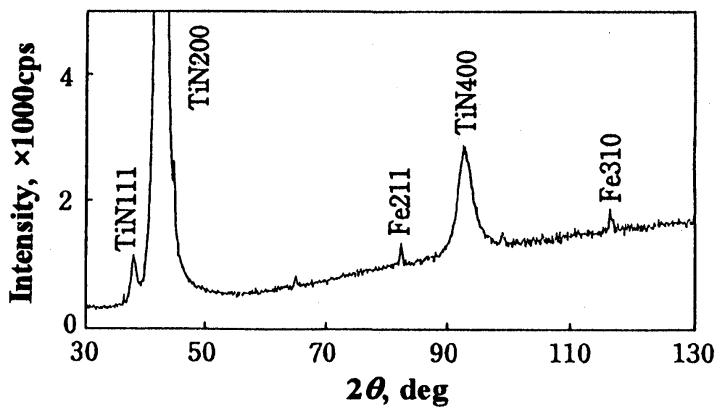

Fig. 19. Diffraction pattern measured on TiN film deposited by dynamic mixing [47].

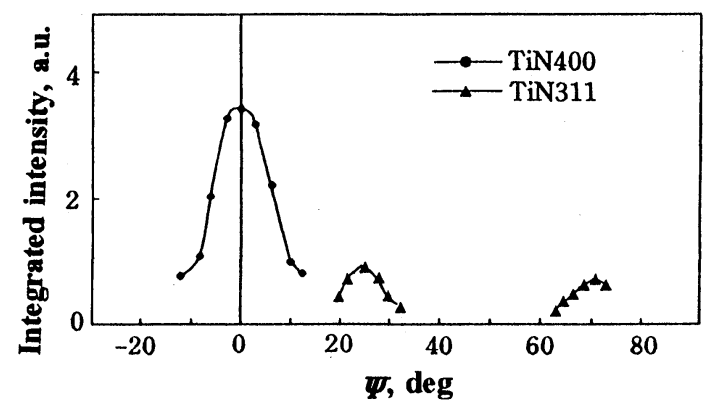

Fig. 20. $\Psi$-angle dependency of 400 and 311 diffraction intensities from TiN film deposited by dynamic mixing [47].

various ceramic coatings (including $\mathrm{TiN}$ ), establishing the relationship between the residual stress and process parameters such as deposition temperatures and other conditions.

Provided here is an example of stress measurement of a TiN film deposited on a spring steel (SUP3) substrate by arc ion plating. Figure 17 shows an X-ray diffraction pattern from the specimen on which $\mathrm{TiN}$ film has been deposited at 523K. Because of strong [111] texture in the film, only two TiN diffraction peaks, 111 and 222, are detected by $2 \theta$ scanning for an as-deposited TiN as well as an annealed TiN film. The results reveal that the peak intensities of 111 and 222 diffraction after annealing are higher than those of the as-deposited TiN film. A more precise measurement of preferred orientation can be made by measuring the integrated intensity of 111 diffraction as a function of $\Psi$. It is shown in Fig. 18 that the intensity peak appears in two narrow regions around $\psi=0 \mathrm{deg}$ and $70.5 \mathrm{deg}$. In this case, residual stresses in TiN film are evaluated by the two-tilt method using 222 diffraction with filtered $\mathrm{CuKa}$ radiation.

The TiN film deposited by dynamic mixing has a different preferred orientation type. In this case, 200 and 400 diffraction peaks are observed as shown in Fig. 19. Figure 20 shows that the TiN 400 peak is restricted within \pm 10 degrees around $\psi=0 \mathrm{deg}$, which means that [100] texture is formed in the film. The observed diffraction peaks around $\psi_{1}=24.2 \mathrm{deg}$ and $\psi_{2}=72.5 \mathrm{deg}$ are obtained from TiN 311 diffraction, which is expected by the angular relation between (100) and (113) planes. In this case, these two diffraction peaks are used for stress evaluation in much the same way as Eq. (6).

Figure 21 shows residual stresses measured in $\mathrm{TiN}$ films deposited by arc ion plating on the surface of a spring steel (SUP3). The as-deposited TiN films have very high compressive residual stresses of $-6 \sim-3.5 \mathrm{GPa}$,

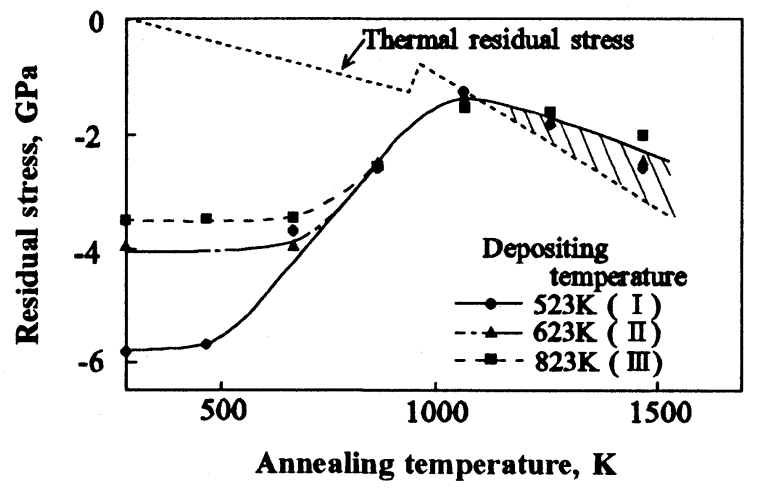

Fig. 21. Residual stress relaxation due to annealing of TiN film deposited on spring steel substrate by arc ion plating [46].

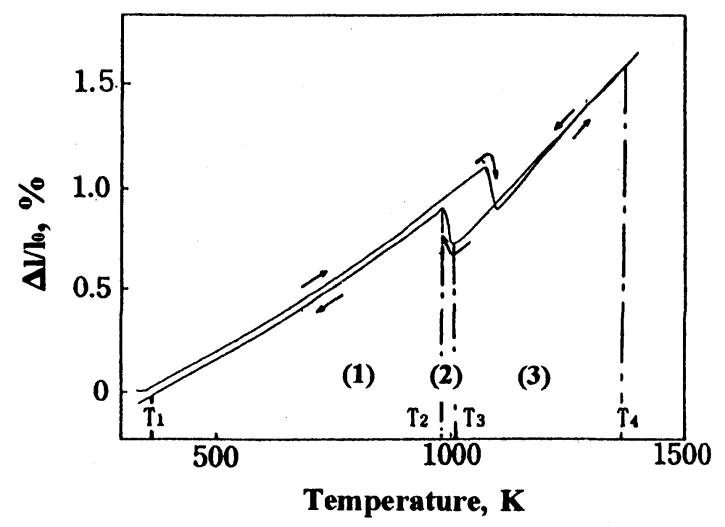

Fig. 22. Thermal expansion and contraction of substrate material, spring steel (SUP3) [46]. 
and the absolute value of residual stress in the TiN film decreases with increasing deposition temperatures. When the specimens are annealed, the compressive residual stress in the TiN film decreases with increasing annealing temperatures, reaching about the same level at $873 \mathrm{~K}$ for all the specimens. After the compressive residual stresses reach the minimum level, about $-1.4 \mathrm{GPa}$ at $1073 \mathrm{~K}$, they increase to the compressive side as the annealing temperature rises.

The development of residual stress in ion plated coatings depends on both the condition of ion bombardment and the deposition environment. In the as-deposited state, ion bombardment of the film during deposition behaves like shot-peening on the film surface at an atomic level. Large compressive residual stresses seem to have been generated by atomic peening during the depositing process.

The thermal residual stress is calculated from Eq. (5), assuming a stress-free state at depositing or annealing temperatures. When the substrate is a carbon steel, a small modification of Eq. (5) is necessary because the thermal expansion coefficient changes with temperature and the volume change occurs because of phase transformation from ferrite to austenite.

Figure 22 is a diagram of thermal expansion and contraction of a substrate material [46]. The cooling process is divided into three regions, i.e., (1) ferrite, (2) transforming and (3) austenite. The average thermal expansion coefficient of ferrite is obtained from the region (1) and that of austenite from the region (3). The substrate material expands during the cooling process, when the structure transforms from austenite to ferrite as shown in the region (2). From the expansion of Eq. (2), the thermal residual stress is represented as follows:

$$
\begin{aligned}
\sigma_{\mathrm{th}} & =\frac{E_{\mathrm{F}}}{1-v_{\mathrm{F}}}\left\{\int_{T_{1}}^{T_{2}}\left(\alpha_{\mathrm{F}}-\alpha_{(1)}\right) \mathrm{d} T\right. \\
& \left.+\Delta \varepsilon+\int_{T_{3}}^{T_{4}}\left(\alpha_{\mathrm{F}}-\alpha_{(3)}\right) \mathrm{d} T\right\}
\end{aligned}
$$

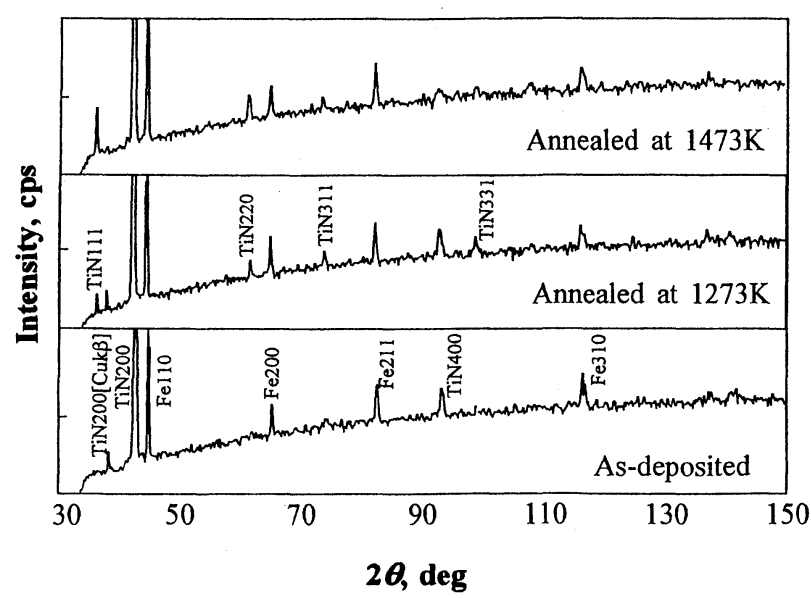

Fig. 23. Diffraction patterns measured on TiN film of as-deposited and of annnealed states [48]. where $E_{\mathrm{F}}(=250 \mathrm{GPa})$ and $\nu_{\mathrm{F}}(=0.19)$ are assumed to be constant, $\alpha_{(1)}\left(=13.9 \times 10^{-6} \mathrm{~K}^{-1}\right)$ and $\alpha_{(3)}\left(=22.4 \times 10^{-6} \mathrm{~K}^{-1}\right)$ are the average thermal expansion coefficient in each region, and $\Delta \varepsilon\left(=1.54 \times 10^{-3}\right)$ is the one-dimensional strain due to volume change. The result calculated from Eq. (9) is represented by the broken line together with the measured values in Fig. 21. The measured and the calculated values for annealing temperatures above $1000 \mathrm{~K}$ are nearly equal but the difference between them increases with increasing annealing temperatures. This difference may arise from stress relaxation caused by plastic deformation in the substrate near the interface or microcracking in the film during the cooling process.

The relaxation of compressive residual stresses by annealing below $1000 \mathrm{~K}$ may be attributed to the relaxation of the initial intrinsic stress generated by ion bombardment.

\subsection{TiC film deposited by thermal CVD method}

TiC film deposited by thermal CVD method shows a [100] texture [48]. Figure 23 shows X-ray diffraction patterns obtained from the TiNcvo film. Because of the high [100] orientation of the film structure, only two intensity peaks, i.e., 200 and 400 diffraction, can be detected in the as-deposited state. When the specimen is annealed at 1273K, TiN 220, TiN 311 and TiN 331 diffraction peaks appear, demonstrating a random orientation in the film. The intensity vs. $\Psi$ curve is similar to that in the former case as shown in Fig. 20. In the present case, the 420 diffraction peaks appear around $\psi_{1}=$ $26.6 \mathrm{deg}$ and $\psi_{2}=63.4 \mathrm{deg}$. With the $2 \theta$ values of these two diffraction peaks, the residual stress is evaluated to be $-1.8 \mathrm{GPa}$.

There is another example of TiC film which is deposited by CVD [19]. In this case, [110] texture is formed in the film, where the relation between the stress and the lattice strain is represented as follows:

$$
\begin{aligned}
\frac{\varepsilon_{33}^{\mathrm{L}}}{\sigma}= & \frac{1}{2}\left(s_{0}^{\mathrm{c}} \cos 2 \gamma+s_{44}^{\mathrm{c}}\right) \sin ^{2} \Psi \\
& +\frac{1}{2} s_{0}^{\mathrm{c}}+2 s_{12}^{\mathrm{c}}
\end{aligned}
$$

where $\boldsymbol{\gamma}$ is a rotation angle of a co-ordinate transformation from the crystal axis represented by [001], [110] and [110] of a single crystal system to the specimen coordinate (see ref. [19]). Since $\gamma$ depends on the diffraction plane used in the measurement, $\varepsilon_{33}^{\mathrm{L}} / \sigma$ does not linearly relate with $\sin ^{2} \psi$ if various diffraction planes are used in the stress evaluation. However, an approximate linear relation is obtained for this texture of TiC film because of its small anisotropy of elasticity (Fig. 24). In convenience, the two-tilt method using 331 diffraction peaks at $\psi_{1}=13.3 \mathrm{deg}$ and $\psi_{2}=49.5 \mathrm{deg}$ or 420 diffraction peaks at $\psi_{1}=18.4 \mathrm{deg}$ and $\psi_{2}=50.8 \mathrm{deg}$ can be adopted. The residual stress of $-2.3 \mathrm{GPa}$ revealed in the film deposited on the SKH51 substrate by CVD is evaluated from 420 diffraction peaks.

As was seen in some examples, the residual stresses in the TiN films deposited by PVD are compressive stresses many times larger than the thermal stress calcu 


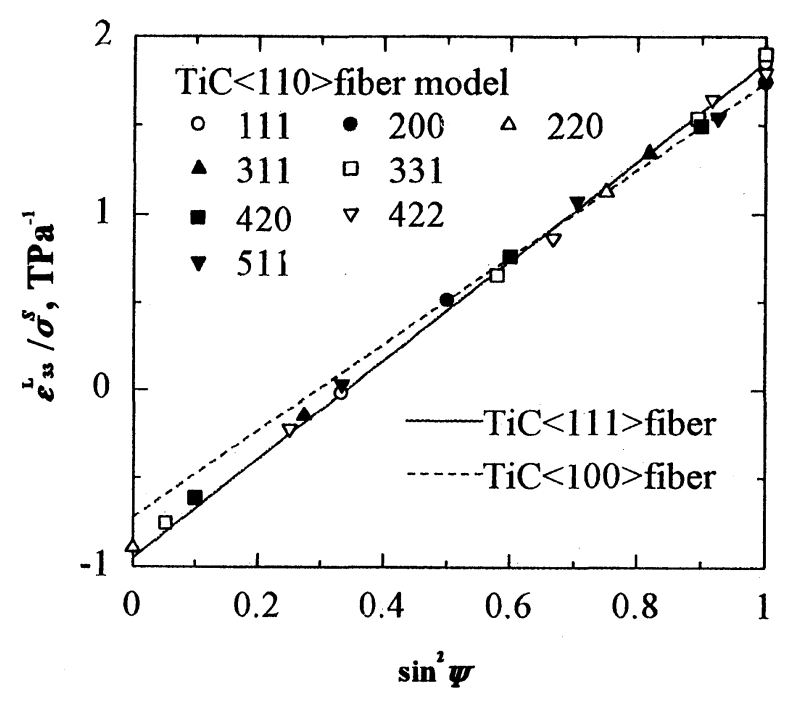

Fig. 24. Calculated lattice strain distribution of [110] preferred oriented TiC film [19].

lated by Eq. (5). However, the compressive residual stresses in the TiN or TiC film deposited by CVD are much smaller than those of PVD cases.

\section{SUMMARY}

The X-ray stress measurement of textured thin films and some application for evaluating the characteristics of film stresses were explained in this paper. High degree of crystal texture appeared in thin hcp and fcc crystalline films deposited by PVD and CVD. A new $\mathrm{X}$-ray stress measuring method was proposed for the measurement of residual stress in these films. By X-ray observation, residual stress state became clear in Al films and patterned $\mathrm{Al}$ lines on a silicon substrate, and TiN and TiC films on a steel substrate. The effect of thermal treatment on the residual stresses was also investigated.

The residual stresses developed in thin films are classified into two types. One is an intrinsic stress and the other an extrinsic stress, with the latter referring to the thermal stress which appeared in the present text and was given by Eq. (5). Namely, the stress was developed by the different rate of thermal contraction between the substrate and the film when the whole system was cooled from the depositing temperature to the room temperature. The intrinsic stress involves all kind of internal stresses excepting thermal stress, such as a compressive stress caused by an ion-peening effect and a tensile stress caused by the effect of vacant spaces on an atomic scale within crystals and/or grain boundaries. In particular, the PVD process develops a very large internal stress in a very thin film. Since these stresses originate on an atomic scale, it is difficult to understand their real effect on high intrinsic stresses.

The X-ray investigation into the film stresses has generated a lot of knowledge on the film/substrate system, but it is not yet sufficient to understand complete characteristics of the film properties. More precise and systematic investigations based on various approaches are necessary to make a full understanding of the film properties.

\section{REFERENCES}

1. V. Hauk, Z. Metallkde., 46 (1955) 33.

2. E. Macherauch and P. Muller, Z. angewandte Physik, 13 (1961) 305.

3. T. Shiraiwa and Y. Sakamoto, The Sumitomo Search, 7 (1972) 109.

4. V. Hauk and G. Vaessen, Z. Metallkde., 76 (1985) 102.

5. H. Matsui, Y. Yoshioka, T. Morooka, K. Hasegawa and T. Nakajima, J. Soc. Mat. Sci., Japan, 36 (1987) 823.

6. T. Hanabusa, B. Scholtes and E. Macherauch, Proc. The 29th Japan Cong. Mat. Res. (1986) 123.

7. T. Hanabusa, M. Tani and H. Fujiwara, J. Soc. Mat. Sci., Japan, 40 (1991) 790.

8. T. Hanabusa, K. Tominaga and H. Fujiwara, J. Soc. Mat. Sci., Japan, 42 (1993) 90.

9. T. Hanabusa, K. Kusaka, K. Tominaga and H. Fujiwara, J. Soc. Mat. Sci., Japan, 42 (1993) 627.

10. K. Kusaka, T. Hanabusa and K. Tominaga, J. Soc. Mat. Sci., Japan, 43 (1994) 799.

11. K. Kusaka, T. Ao, T. Hanabusa and K. Tominaga, Thin Solid Films, 332 (1998) 247.

12. T. Hanabusa, H. Hosoda, K. Kusaka and $K$. Tominaga, Thin Solid Films (to be published).

13. K. Kusaka, T. Hanabusa, M. Nishida and F. Inoko, Thin Solid Films, 290-291 (1996) 248.

14. T. Hanabusa, K. Kusaka, M. Nishida and F. Inoko, The Fifth International Conference on Residual Stresses (ed. by T. Ericsson, M. Oden and A. Andersson), Institute of Technology, Linköping University, Linköping, Sweden (1998) 854 .

15. K. Tanaka, K. Ishihara and K. Inoue, J. Soc. Mat. Sci., Japan, 45 (1996) 1138.

16. T. Matsue, T. Hanabusa and Y. Ikeuchi, J. Soc. Mat. Sci., Japan, 44 (1995) 1121.

17. J.A. Sue, Surf. and Coat. Technol., 54/55 (1992) 154.

18. R. Cerny, R. Kuzel, Jr., V. Valvoda, S. Kadlec and J. Musil, Surface and Coatings Technology, 64 (1994) 111.

19. S. Ejiri, Z. Lin, T. Sasaki and Y. Hirose, J. Soc. Mat. Sci., Japan, 46 (1997) 750.

20. M.T. Duffy, C.C. Wang, G.D. O'Clock Jr., S.H. McFarlane III and P. J. Zanzucchi, J. Electron. Mater., 2 (1973) 395.

21. J.K. Liu, K.M. Lakin and K.L. Wang, J. Appl. Phys., 46 (1975) 3730.

22. T. Shiosaki, T. Yamamoto, T. Oda and A. Kawabata, Appl. Phys. Lett., 36 (1980) 643.

23. M.T. Wauk, and D.K. Winslow, Appl. Phys. Lett., 13 (1969) 286.

24. A.J. Schskus, T.M. Reeder and E.L. Paradis, Appl. Phys. Lett., 24 (1974) 155.

25. Y. Murayama, K. Kashiwagi and M. Kikuchi, J. Vac. Technol., 17 (1980) 796.

26. K. Tominaga and Y. Shintani, Japanese Jnl. Appl. Phys., 28, Supplement (1989) 7.

27. J. Fukura and H. Fujiwara, J. Soc. Mat. Sci., Japan, 32 (1966) 1597.

28. C.J. Shute, J.B. Cohen and D.A. Jeannotte, Mater. Res. Soc. Symp., Proc., Vol. 130, Materials Research Society, Pittsburgh (1989) 29. 
29. P.A. Flinn and C. Chiang, J. Appl. Phys., 67 (1990) 2927.

30. S.L. Lee and G.P. Capsimalis, Adv. in X-Ray Anal., 39 (1997) 257.

31. K. Tanaka, K. Ishihara and K. Inoue, J. Soc. Mat. Sci., Japan, 45 (1966) 945.

32. K. Tanaka, K. Ishihara, Y. Akiniwa and H. Ohta, Mat. Sci. Res. Int., 2 (1996) 153

33. P.S. Ho, L-S. Yeo, S.G.H. Anderson and C.K. Hu, AIP Conference Proceedings, No. 305 (ed. by P.S. Ho, C-Y. Li and P. Totta), American Institute of Physics, New York (1994) 62.

34. M. Schneegans, G.M. Zorn, AIP Conference Proceedings No. 305 (ed. by P.S. Ho, C-Y. Li and P. Totta), American Institute of Physics, New York (1994) 111.

35. R. Venkatraman, P.R. Brennan and J.C. Bravman, Mater. Res. Soc. Symp. Proc., Vol. 239, Materials Research Society, Pittsburgh (1992) 227.

36. T. Onishi, E. Iwamura, K. Takagi and K. Yoshikawa, Kobe Steel Eng. Rep., 48 (1998) 29.

37. Y. Kawano, H. Shinriki, T. Ohta and M. Katayama, Proc. 29th Symp. X-Ray Studies on Mech. Behav. Mater., Kyoto (1992) 45.

38. K. Tanaka, Y. Akiniwa, K. Inoue and H. Ohta, The Fifth International Conference on Residual Stresses (ed. by $\mathrm{T}$. Ericsson, M. Oden and A. Andersson), Institute of Tech- nology, Linköping University, Linköping, Sweden (1998) 874.

39. A.J. Perry, M. Jagner, W.D. Sproul and P.J. Rudnich, Surf. and Coat. Technol., 42 (1990) 49.

40. M.K. Hobbs, R.G. Cooke, B.Harris and H. Reiter, British Ceram. Proc., 39 (1989) 119.

41. G.C. Chang and W. Phucharoen, Surf. and Coat. Technol., 32 (1987) 307.

42. J.A. Sue, Surf. and Coat. Technol., 54/55 (1992) 154

43. S.J. Bull, A.M. Jones and A.R. McCabe, Surf. and Coat. Technol., 54/55 (1992) 173.

44. W.G. Sloof, B.J. Kooi, R. Delhez, Th.H. de Keijser, and E.J. Mittemeijer, J. Mater. Res., 11 (1996) 1440.

45. T. Matsue, T. Hanabusa and Y. Ikeuchi, Thin Solid Films, 281-282 (1996) 344.

46. T. Matsue, T. Hanabusa and Y. Ikeuchi, The Fifth International Conference on Residual Stresses (ed. by $T$. Ericsson, M. Oden and A. Andersson), Institute of Technology, Linköping University, Linkőping, Sweden (1998) 874.

47. T. Matsue, T. Hanabusa and Y. Ikeuchi, J. Soc. Mat. Sci., Japan, 45 (1996) 1119.

48. T. Matsue, T. Hanabusa and Y. Ikeuchi, Mater. Sci. Res. Int., 5 (1999) 45.

\section{Book Review}

\section{"UNDERSTANDING MATERIALS SCIENCE: History- Properties- Applications"}

Author: Hole E. Bummel, Professor, Department of Materials Science and Engineering, University of Florida Publisher: Springer-Verlag New York, 1998, 407pages, 88DM, ISBN:0-387-98303-1

Reviewer: Tatsuo Inoue, Department of Energy Conversion Science, Kyoto University

The phrase "understanding the history of materials means understanding the history of mankind and civilization" by the author presented on the spread page is sympathized by the present reviewer, who is also interested in the history of materials, especially, steel and swords made of steel. Indeed, materials themselves and the properties are developed along with the history of mankind. So, it is attractive for the students and engineers in the primary stage of fundamentals of material science to pileup the knowledge of the history and related social matters.

The level of the book is not so high since the monograph is written as a text book for the primary course. However, it covers wide range of material sciences which the readers of this journal, MSRI or the member of the Society already know the each part of content consisting three parts. The first and second parts treat the mechanical and electronic properties with the clear description of quantum mechanics, atomic structure of iron and metallic bonding, crystallography, dislocation theory, phase diagram of alloys, dielectric property, paramagnetizm as well as mechanics of materials for many kind of materials including metals, glass, ceramics cement, wood, rubber, polymer, and so on. In these parts, some sections are sheared for the history of materials in the chronological order such as ages of stone, copper-stone, bronze, iron followed by the age of modern electronic materials and some critical descriptions of ceramics age with colored photos of antique metal crafts as well as some illustrations.

The final part covers the materials and world where the author tries to emphasize his own opinion on the recent development of economics and environmental problems.

The reviewer strongly recommends this book to the experts in this field as well as the beginners for global understanding of the material science. 